\title{
Othmar Plöckinger (éd.), Quellen und Dokumente zur Geschichte von Mein Kampf 1924-1945
}

\section{François Delpla}

\section{OpenEdition}

\section{Journals}

Édition électronique

URL : http://journals.openedition.org/ifha/8578

DOI : $10.4000 /$ ifha. 8578

ISSN : 2198-8943

\section{Éditeur}

IFRA - Institut franco-allemand (sciences historiques et sociales)

\section{Référence électronique}

François Delpla, «Othmar Plöckinger (éd.), Quellen und Dokumente zur Geschichte von Mein Kampf 1924-1945 », Revue de I'IFHA [En ligne], Date de recension, mis en ligne le 01 février 2017, consulté le 24 septembre 2020. URL : http://journals.openedition.org/ifha/8578; DOI : https://doi.org/10.4000/ifha. 8578

Ce document a été généré automatiquement le 24 septembre 2020.

(C)IFHA 


\section{Othmar Plöckinger (éd.), Quellen und Dokumente zur Geschichte von Mein Kampf 1924-1945}

François Delpla

\section{RÉFÉRENCE}

Othmar Plöckinger (dir.), Quellen und Dokumente zur Geschichte von Mein Kampf 1924-1945, Stuttgart: Franz Steiner Verlag (Beiträge zur Kommunikationsgeschichte 28), 2016, 695 p., $99 €$ 
Ce livre est un produit dérivé de la réédition critique de Mein Kampf parue au début de 2016, confectionné par l'un de ses principaux auteurs. Il s'agit d'un pur recueil de documents, sans le moindre commentaire (à l'exception, de loin en loin, d'un point d'exclamation quand Othmar Plöckinger trouve une formulation ou une assertion amusante: une concession, sans doute, à l'obligation généralement ressentie de nos jours d'éditer les œuvres nazies en précisant qu'on n'y souscrit pas). Il rassemble d'une part les principales archives qui éclairent la rédaction du livre (surtout de son premier tome, en 1924, pendant l'incarcération de Hitler) et ses différentes éditions, d'autre part des appréciations sur son contenu, soit publiées, soit émises

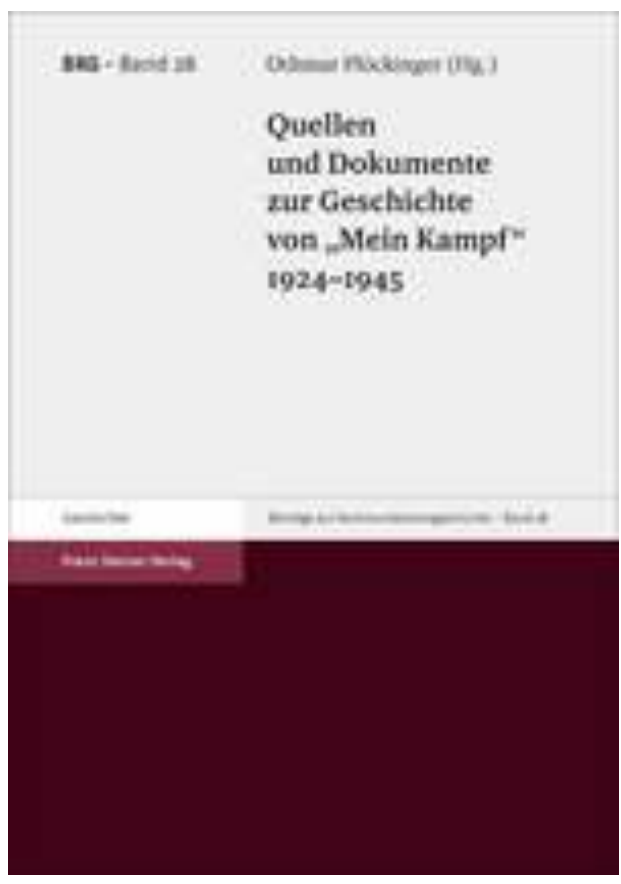
en privé, jusqu'en 1945. Certains de ces documents ont été utilisés dix ans plus tôt dans le premier ouvrage de l'auteur, Geschichte eines Buches. Adolf Hitlers Mein Kampf 1922-1945 (Oldenburg, 2006) et d'autres cinq ans plus tard, dans une réédition enrichie. Cette dernière fait l'objet de nombreux renvois en note.

La première section reproduit exhaustivement les matériaux disponibles sur la genèse de Mein Kampf, la seconde les comptes de l'éditeur à son sujet jusqu'en 1933. La troisième comporte une cinquantaine de recensions de toutes tendances échelonnées de 1925 à 1932, la quatrième (la plus volumineuse) des textes ayant trait à l'ouvrage, toujours avant 1933, la cinquième et dernière rendant compte des usages du livre entre 1933 et 1945.

Une telle richesse défie le résumé et rend difficile un florilège. Cependant, le propos de l'auteur n'est pas seulement de fournir des matériaux pour préciser les détails de l'élaboration de la Bible nazie et de sa réception, mais aussi de récuser bon nombre d'idées fausses, encore bien ancrées, sur ces sujets. Et avant tout le préjugé selon lequel le livre était unlesbar und unlesen, illisible et non lu. O. Plöckinger prend ainsi un malin plaisir à citer un critique littéraire bien connu, productif avant, pendant et après le Troisième Reich, Paul Fechter, qui, dans l'édition de 1941 de son histoire de la littérature allemande, définit et vante une révolution stylistique nazie apte à passionner les lecteurs. Quelques recensions vont dans le même sens en parlant d'une prose entraînante par le fait même qu'elle a visiblement été prononcée avant d'être écrite.

Trois lettres de Rudolf Hess ruinent les fables selon lesquelles le futur lieutenant du Führer aurait pris une part prépondérante dans la rédaction du premier tome, qu'il aurait dactylographié sous la dictée. Hitler s'isolait pour écrire, tapait lui-même et Hess, qui lui apportait régulièrement du thé, était tout heureux quand il le retenait pour lui lire tout ou partie d'un chapitre. Quant au deuxième tome, il a été dicté à diverses secrétaires, dont l'une, Hertha Oldenburg, fait l'objet d'un document ( $\left.n^{\circ} 37\right)$. La 
légende de la collaboration Hess-Hitler est d'origine nazie : plusieurs documents datés de 1933 et 1934 font accroire que Hess a tapé tout le premier tome au cours de longues séances d'isolement à deux dans la cellule du chef. On peut donc, en rapprochant tous ces éléments, préciser un point non négligeable de l'histoire du nazisme : dès la prise du pouvoir, la propagande fait artificiellement de Hess un apôtre élu de longue date et une sorte d'alter ego du Führer, dont lui seul reçoit le titre de "suppléant" (Stellvertreter). Ces efforts de propagande accompagnent l'accession de Hess à la tête du parti nazi, dont le chancelier, tout en conservant la direction nominale, lui abandonne les rênes le 21 avril 1933. Les futurs biographes devront en tenir compte, ainsi que les historiens qui traiteront de la préhistoire du fameux vol vers l'Écosse, en mai 1941.

O. Plöckinger déchire à belles dents un mythe du même ordre, quoique différent dans ses suites, quand il dément toute participation de Bernhard Stempfle (1882-1934) à l'écriture du livre - la démonstration se trouvant dans son livre de 2006. L'homme, qui n'avait pas été jésuite mais hiéronymite, était détaché de toute fonction religieuse depuis le début des années 1920 pour n'être plus qu'un professeur et un journaliste völkisch. Son meurtre pendant la Nuit des longs couteaux, sur les causes duquel les bruits les plus divers continuent de courir, ne pourra plus être attribué à la honte que Hitler aurait ressentie d'avoir vu son allemand corrigé par l'ancien moine. Lequel publie en plusieurs fois en 1925, dans un journal qu'il dirige, l'une des premières recensions de Mein Kampf et l'une des plus longues. Il exprime des désaccords sur la question monarchique et sur celle, cruciale pour Hitler, du Sud-Tyrol, mais c'est peutêtre plus par de discrets persiflages qu'il a pu déplaire et sceller son destin, notamment lorsqu'il relève que Hitler fait de sa ville natale de Braunau un nouveau Bethléem.

Parmi les recensions, on peut constater que celles des journaux de gauche font une large place à l'antisémitisme en y voyant le fil rouge de la pensée de l'auteur. Ainsi, la phrase aujourd'hui fameuse sur les 10000 notables juifs qu'il aurait fallu gazer en 1914 pour affermir la communauté nationale n'est pas passée inaperçue à l'époque. Sur un projet similaire, le 21 février 1927 (document 68), le périodique Abwehr-Blätter, l'organe de la Ligue de défense contre l'antisémitisme, porte un jugement significatif : lorsque Hitler déclare qu' un jour, un tribunal national allemand aura à juger et à faire exécuter quelques dizaines de milliers d'organisateurs responsables de la trahison de novembre [1918] et de tout ce qui s'y rapporte ", le recenseur juge qu'une telle phrase relève de la psychiatrie. Hitler est donc fou, mais seulement par moments, et un fou pas vraiment dangereux, tant son appétit de meurtre semble irréaliste. Mais toute la mouvance völkisch ne se laisse pas embarquer aussi facilement. Un professeur de philosophie, Arnold Paul Ruge (1881-1945), émet des objections qu'il estime „, non dépourvues d'importance ». Sur la vision hitlérienne du marxisme tout d'abord. Hitler $\mathrm{y}$ voit une manœuvre juive pour détourner les ouvriers de la nation, Ruge, tout au contraire, une ruse d'une "aristocratie juive " pour s'inféoder ces mêmes ouvriers ; cette aristocratie, c'est-à-dire la bourgeoisie juive, a intérêt à la guerre et, pour mieux la provoquer et l'entretenir, fait des ouvriers des patriotes, en les endoctrinant par la presse, en les achetant et en les conduisant au massacre, dans les guerres et les révolutions. Selon Ruge, il ne faut donc pas s'appuyer sur les masses, mais les soumettre à un contre-endoctrinement dans lequel Mein Kampf, souhaite l'auteur, tiendra une place de choix. Enfin Ruge, décidément soucieux de réhabiliter la bourgeoisie, réprouve les jugements de Hitler sur sa pusillanimité et la félicite d'avoir engendré les théoriciens antisémites allemands des dernières décennies. Bref, avec 
Arnold Paul Ruge, l'élitisme, l'intellectualisme et la candidature des vieilles classes dirigeantes à la direction du „, redressement national » font de la résistance !

Certaines recensions sonnent au contraire comme un ralliement, par exemple celle d'Adolf Bartels (1862-1945): l'un des plus célèbres écrivains völkisch conclut une recension entièrement élogieuse par l'idée que „l'avenir de l'Allemagne est impossible » sans la lecture de ce livre par „tous les hommes sérieux ».

Quant aux rapports entre Hitler et la religion chrétienne, ils font l'objet d'un article de revue daté d'avril-mai 1931 dû au journaliste catholique bavarois Peter Langendorf (document 109). Il s'agit du document le plus long, bien qu'il soit amputé de ses cinq derniers chapitres. Il y a là un socle doctrinal fondant l'attitude plutôt ferme de l'épiscopat cette année-là et jusqu'à la fin de mars 1933, marquée notamment par l'interdiction faite aux baptisés d'adhérer au NSDAP. Ainsi la levée de l'interdit décidée en mars 1933 par la conférence des évêques (ouvrant la voie au concordat signé en juillet et au suicide du parti catholique Zentrum) ne pourra plus être sérieusement attribuée à une naïve ignorance des conceptions de Hitler ni de ses projets par quiconque prend en compte cette revue de détail des incompatibilités entre nazisme et christianisme. L'Église a bel et bien joué les apprentis sorciers en croyant avoir affaire à un automate et en étant convaincue de pouvoir le maitriser grâce au poids théorique des conservateurs dans le gouvernement.

Sur la diffusion du livre après 1933, une lettre (document 147) de Carl Goerdeler, maire de Leipzig, à son collègue de Francfort le 25 mai 1936 permet de constater que la distribution de Mein Kampf aux jeunes mariés ne faisait l'objet que d'une circulaire incitative du ministère de l'Intérieur, permettant aux municipalités de se dérober en invoquant des difficultés financières... et beaucoup le faisaient, d'autant plus que les éditions Eher refusaient de fournir pour l'occasion des exemplaires bon marché. Voilà qui dément un autre préjugé (celui de la distribution automatique du livre aux jeunes ménages) et qui incite à réfléchir au doigté dont faisait preuve à l'occasion le totalitarisme nazi : sa propagande ne faisait pas, comme dans d'autres dictatures, l'objet d'une avalanche aveugle de papier imprimé ; Hitler, à supposer (ce qui est probable) qu'il ait été consulté sur ces dispositions et par le ministre de l'Intérieur Wilhelm Frick et par son ami Max Amann, directeur des éditions Eher, préférait la qualité à la quantité.

Mis à part le futur résistant Goerdeler, une autre célébrité a droit à la publication d'une lettre (document 115) : Martin Heidegger, qui enjoint à son frère Fritz le 18 décembre 1931 de se colleter (auseinandersetzen) avec le livre, tout en remarquant que ses premières pages autobiographiques sont „faibles" (mais 0. Plöckinger indique malicieusement en note que Himmler avait émis un jugement du même ordre dans son journal en 1925). Le philosophe vante "l'instinct politique exceptionnel et sûr " de l'auteur qui lui avait permis de voir clair "quand nous étions encore tous dans le brouillard». Il prédit avec une satisfaction visible que le mouvement nazi va encore beaucoup grandir et indique qu' 'il ne s'agit plus de petites querelles de partis mais du sauvetage ou du déclin de l'Europe et de la culture occidentale». La lettre étant destinée à envoyer des vœux de Noël, le prédicateur éprouve le besoin d'ajouter une phrase encore plus intéressante: „, Les réflexions sur ces matières ne détruisent pas la paix de Noël, mais nous ramènent à l'Être et aux devoirs des Allemands, c'est-à-dire là même où la forme de cette merveilleuse fête trouve son origine.» 
Terminons ce tour d'horizon par un passage du journal de Wolfram von Richthofen (document 169), cousin du „, baron rouge » abattu pendant la Première Guerre mondiale et aviateur comme lui (il avait dirigé le bombardement de Guernica en 1937). En ce 6 janvier 1942, il commande la $4^{\mathrm{e}}$ flotte aérienne, engagée en Ukraine orientale et aux prises avec de dures contre-attaques soviétiques. L'officier se ressource dans la prose du commandant suprême: „Lis encore une fois dans le Kampf le chapitre sur la politique extérieure et orientale. Tout de même très intéressant et répondant, $\mathrm{y}$ compris dans la situation actuelle, quasiment à toutes les questions. Je vais veiller à ce qu'on mette davantage l'accent là-dessus auprès de la troupe, dans tout mon ressort. »

Mis à part, donc, les points d'exclamation bien superflus (qui ne sont pas les plus forts du livre!), nous avons là une illustration éclatante de la nécessité et de l'utilité du travail approfondi mené par une nouvelle génération d'historiens sur Mein Kampf sous l'aiguillon des débats engendrés par l'arrivée des proses hitlériennes dans le domaine public en 2016.

INDEX

Index chronologique : Époque contemporaine

Thèmes : Sources, Outils de travail, Histoire des idées 